\title{
A Comparative Study on Distributed Location Management Strategies in Wireless Networks
}

\author{
Hoang Nguyen-Minh, Harmen R. van As \\ Vienna University of Technology, Institute of Communication Networks \\ Favoritenstrasse 9/388, A-1040, Vienna, Austria \\ Tel.: + 43-1-58801-38815, Fax: + 43-1-58801-38898 \\ Email: Hoang.Nguyen-Minh@tuwien.ac.at
}

Keywords: $\quad$ TINA, UMTS, Distributed Location Management.

Abstract: Location management is an essential process in future mobile communication networks. An important issue is an efficient management of the location database. In this paper, the next generation mobile communication networks are proposed to integrate with a TINA-compliant architecture enabling to handle that kind of mobile-specific processes. We consider a distributed location database architecture for location management performing the following strategies: HLD (home location database only), HLD-VLD (HLD with a visited location database), or VLD-CLD (HLD and VLD with a cache location database). This paper discusses design, modelling and the comparison of the mentioned distributed location management strategies. The performance measures used for comparison are communication cost (signalling messages), computational cost (database accesses) and average total cost. For the performance analysis, we assume that the cost of updating a cache pointer and a user profile at CLD/VLD is equal. Results show that the combination of replication with caching scheme (VLD-CLD) performs better than the replication scheme (HLD-VLD) for a very wide range of call-to-mobility ratio (CMR).

\section{INTRODUCTION}

Location management is an essential process in mobile communication networks supporting location independent communications to users [1]. Location management is concerned with the issues of tracking and finding the 
mobile terminals (MT) in order to allow roaming within the network coverage area. The third generation mobile communication network, Universal Mobile Telecommunication System (UMTS), has adopted the functional type architecture of Intelligent Networks (IN) and distributed database architecture has been proposed [2]. It is still an open question whether the designated concepts of IN will be sufficient to provide the required powerful mechanisms needed for service provisioning, connection management, as well as mobility management in multi-service provider domains. TINA (Telecommunications Information Networking Architecture) proposes a framework for future telecommunication systems based on distributed and object-oriented computing that enables easy implementation of a distributed database in a distributed processing environment for location management.

Three strategies for location management in wireless networks are proposed in this contribution: HLD (home location database only), HLD-VLD (HLD with a visited location database), and VLD-CLD (HLD and VLD with a cache location database). We give the protocols of location updating/registration and call delivery procedures for these distributed databases. We compare those algorithms and show the effectiveness of the algorithm of VLD-CLD and HLD-VLD schemes by an analytical method.

The rest of the paper is organized as follows. In Section 2, we describe the system model and the distributed location database architecture. In Section 3, we give some of the strategies for location update and call delivery. We present an analysis of these algorithms and compare their performance in Section 4. Finally, some concluding remarks are given in Section 5.

\section{SYSTEM MODEL}

The basic system model for wireless communication networks using a TINA-compliant model, similar to the one presented earlier [5], is shown in Figure 1. Essentially, each cell has a base transceiver station (BTS) to which the mobile terminals of the cell communicate through a wireless link. Each BTS is connected to a mobile switching center (MSC) through a wired network. The BTSs and MSCs are modelled as sub-networks and layer networks that are under the control of the layer network domains (LND) and the connectivity provider domain. The cells are aggregated into contiguous geographical regions called Location Areas (LA). An LA is the granularity at which the network keeps track of the locations of the MTs. An object located in the MT called Terminal Location Management (TLM) performs location updating [5]. Each MSC has an object called Location Manager (LM), which performs the location update/registration and search procedures. The LM has access to the home location database (HLD) within the LND, which is used 
to store location and service information for each registered user of the wireless network. Each MSC has a cache location database (CLD) or a Visited location database (VLD) performing the schemes of caching or replication. The CLD/VLD also plays a role in handling call control information, authentication and billing. Note that a CLD/VLD has a geographical dependency and is coupled to an MSC and a fixed set of BTSs (grouped into one or several LAs). The CLD and VLD architectures can also be generalized into a distributed database design. In this distributed design, user profiles are partitioned and stored in multiple databases at different physical locations within the MSC. A TINA-compliant wireless network can support a distributed processing environment (DPE), a middleware kernel like CORBA's ORB, on which the computational objects are running and interacting.

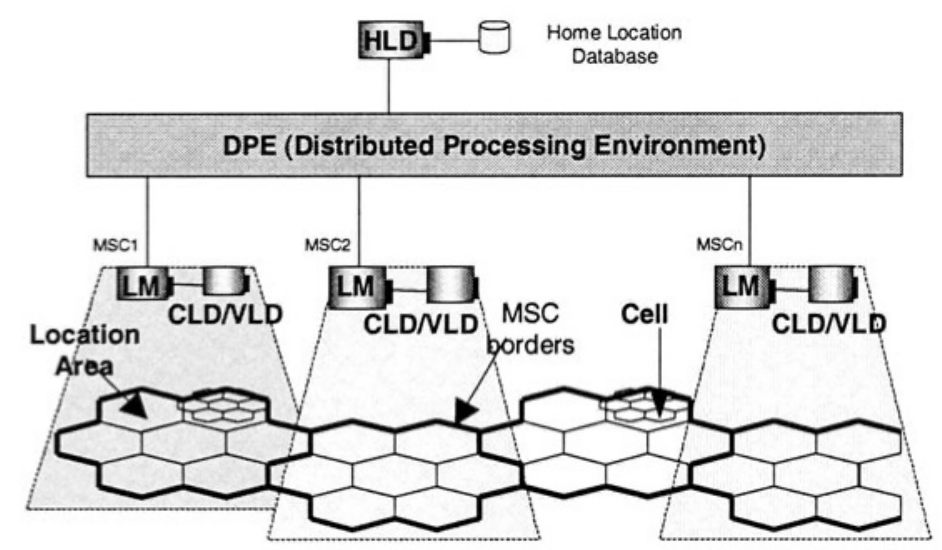

Figure 1. Wircless Network Architecture based on TINA-compliant model

\section{LOCATION MANAGEMENT PROTOCOLS}

\subsection{HLD-only}

In this strategy, only the home location database (HLD) keeps the location information of its enrolled MTs. Thus, one database query is enough to deliver the call. Whenever an MT moves to another LA, a location update request is delivered only to its home HLD to update the current LA address. For the call delivery procedure, the location information of a called MT is retrieved from the called home database to determine the current LA address and a paging process is started. This strategy reduces the number of data- 
base accesses and simplifies the call delivery procedure [3]. The location update procedure and call delivery procedure are given in Figures $2 \mathrm{a}$ and $2 \mathrm{~b}$, respectively.

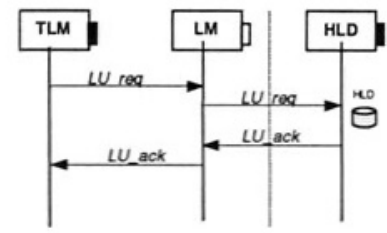

(a) Location update

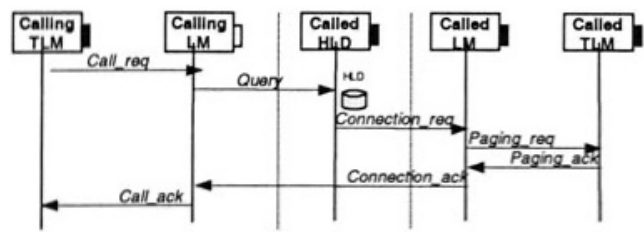

(b) Call delivery

Figure 2. HLD-only location management

\section{$3.2 \quad$ HLD-VLD}

In this section, we introduce a simple replication (SR) scheme called HLD-VLD. It is based on distributed visited location databases (VLDs) with a replication function that enables copying the user information from the HLD to the VLD in the visited MSC corresponding to the user's movement. The VLD temporarily stores the user profiles, and the location information (the current LA) of MTs roaming in its area. The HLD permanently stores the user profiles and points to the VLD that is in charge of the LA in which the MT is currently roaming.

\subsubsection{Location Update (LU)}

When an MT leaves an LA and enters another LA, it initiates a location update procedure. There are two types of location update procedure: intraMSC and inter-MSC.

\section{- Intra-MSC LU}

When an MT enters a new LA in the same MSC, the updating procedure will be done locally at the current VLD of the MSC, as shown in Figure $3 \mathrm{a}$. The cost due to the location update can be reduced because the MT does not need to register at its home HLD.

\section{- Inter-MSC LU}

This procedure will be triggered whenever the MT enters a new LA in the new MSC as shown in Figure 3b. The LM determines that it is an inter-MSC procedure by the identifier of the MSC, and then forwards the request to the home location database at the HLD. The new location in- 
formation of the MT is updated in the HLD, and if authentication successful is, an acknowledgement of the update request is returned to the current VLD. The old VLD is also informed to remove the record of the MT and return the acknowledgement message to the HLD.
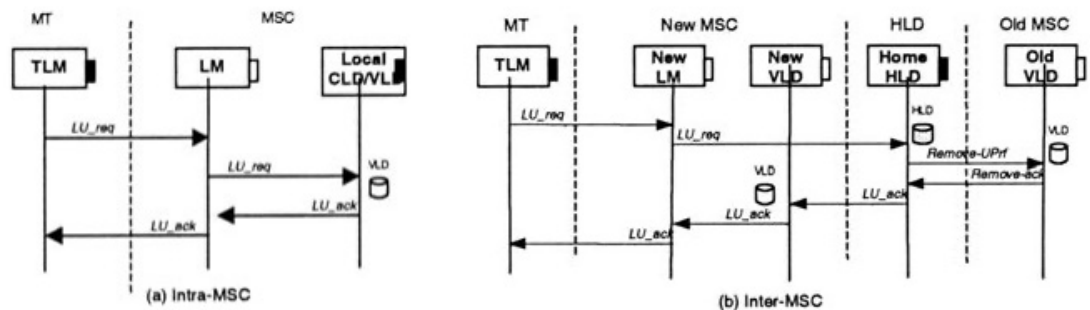

Figure 3. HLD-VLD location update procedure

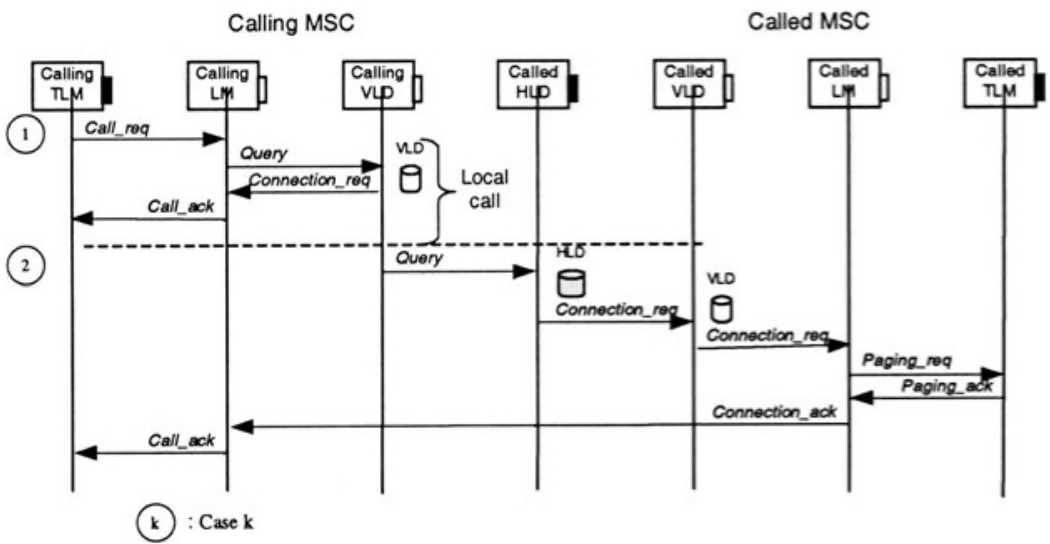

Figure 4. HLD-VLD call delivery procedure

\subsubsection{Call delivery procedure}

For the call delivery protocol, the location information of a called MT is first retrieved from the VLD of the calling MT to check whether the called and calling parties are in the same MSC. If so, it is a local call delivery and the call will be routed without any HLD access (case 1 in Figure 4). If not, the HLD is queried for the location information of the called MT. The HLD contains a pointer to the called VLD in whose associated LA the called MT is currently located, and launches the query to that VLD. The VLD in turn queries the called LM to determine whether the user terminal is capable of receiving the call (by performing a paging procedure). The called LM returns a routable address back to the calling LM. At this point the connec- 
tivity provider domain can route the call by the connection procedure which is in detail described in [5].

\subsection{VLD-CLD}

In this section, we introduce a simple replication with caching (SRC) scheme called VLD-CLD. Each MSC is equipped with a visited location database (VLD), as mentioned in the previous section and a local cache location database (CLD) that maintains temporally location caching of called MTs originated from their MSC. Location caching for a given user should be employed if a large number of calls originate for that user from that MSC, related to the user's mobility. The cache information is kept at the CLD/MSC from which the calls originate. Location caching involves the storages of location pointers at the originating MSC that point to the VLD called the pointed VLD (and the associated MSC) where the MT is currently registered.

\subsubsection{Location update}

The location update procedure for this scheme is similar to the HLDVLD scheme in Section 3.2.1.

\subsubsection{Call delivery procedure}

Each MSC equipped with a cache location database (CLD) that maintains temporarily pointers to pointed VLDs and a visited location database (VLD) that stores the user profile replicas of users visited in its LA. Note that the cache information is kept at the calling CLD/MSC from which calls originate. On receipt of a call request (call_req) message, the calling LM checks its local VLD whether the information of the called party is available locally.

If the calling party and the called party are in the same MSC, and the called user profile is stored at the VLD, we have a local call procedure as shown in case 1 of Figure 5. In the local call, the called user location is received locally at the VLD; hence it completes a call fast and needs less signaling traffic and fewer database accesses. This is useful due to the fact that many call patterns in personal communication services today have the locality between the calling and called parties.

A remote call delivery situation occurs when the calling and called parties are in different MSCs. The calling LM then checks the local CLD for location information of the called MT. If a cache entry exists and the pointed VLD is queried, two situations are possible. If the called MT is still registered at the LA of the pointed VLD, we have a hit case in Figure 5 (case a). 
The called MT is paged and the routing address is returned to the calling LM. Otherwise, the pointed VLD returns a cache miss in which the local CLD queries the HLD of the called party and then caches the location information of the called MT (the pointer to its pointed VLD) as shown in case $b$ of Figure 5.

Calling MSC

Called MSC

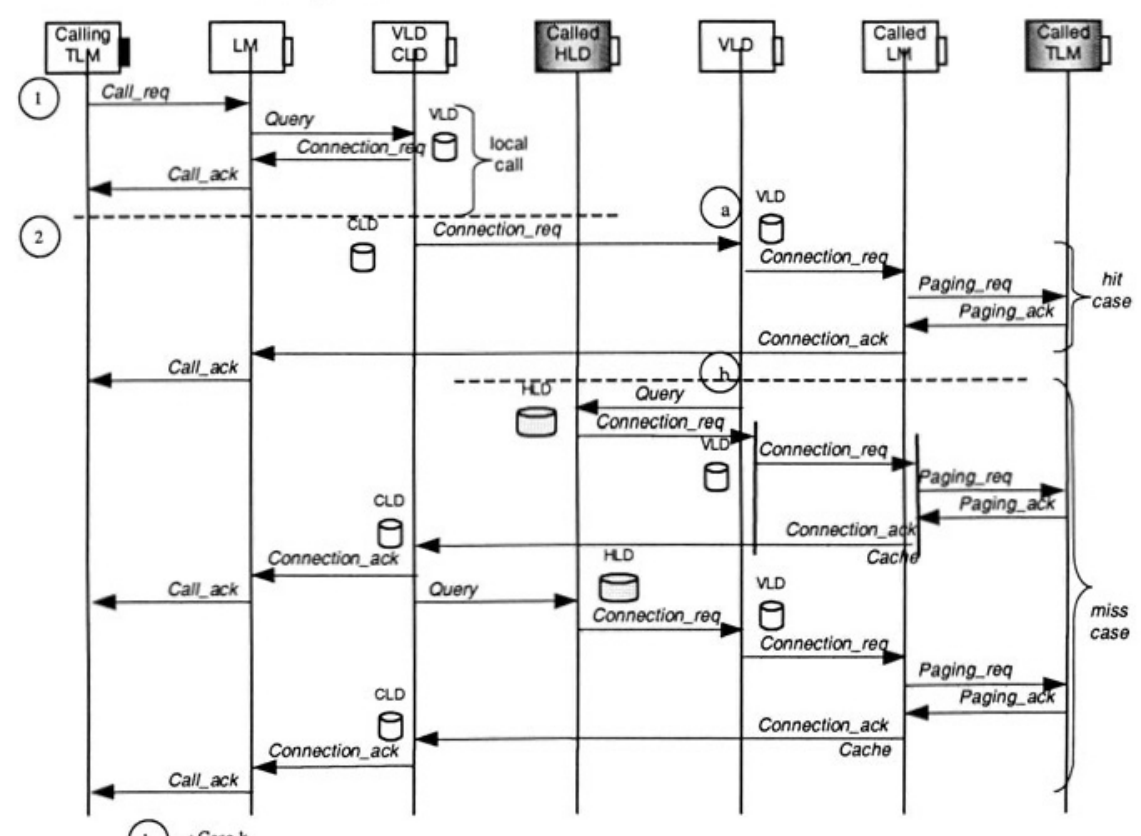

Figure 5. SRC (VLD-CLD) call delivery procedure

\section{PERFORMANCE ANALYSIS}

\subsection{System Analysis}

We assume that the density of users is uniform throughout the area, that the direction of motion with respect to the border is uniform on $[0,2 \pi)$, and that all the cells are of the same shape and size and together form a contiguous area. Let $t_{c}$ and $t_{m}$ be independent and identically distributed random variables representing the call arrival time and the LA residence time. We assume $t_{c}$ and $t_{m}$ to be exponentially distributed with the rate of $\lambda_{c}$ and $\lambda_{m}$, respectively. According to [7], the border-crossing rate out of a circular LA 
and MSC areas, denoted by $v$ and $\mu$, with $v$ is MT's average speed, are given by

$$
v=\frac{\pi v}{4 r_{L A}} \text {, and } \mu=\frac{\pi v}{4 r_{u}}
$$

where $r_{L A}$ and $r_{M S C}$ are the radius of the circular area of the LA and the MSC accordingly. Note that an MT that crosses an MSC will also cross an LA border. So, the border-crossing rate for which the MT still stays in the same MSC

$$
\lambda=v-\mu=\frac{\pi v}{4}\left(\frac{1}{r_{u}}-\frac{1}{r_{M S C}}\right)
$$

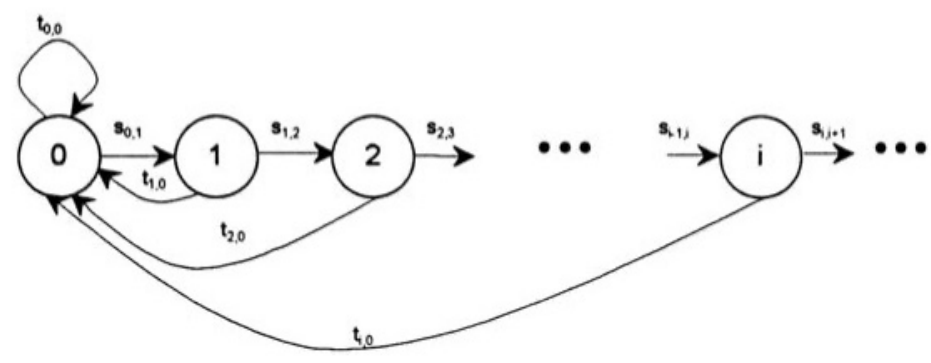

Figure 6. Imbedded Markov chain model

Figure 6 shows an imbedded Markov chain model, which describes the location update process of an MT. The state of the imbedded Markov chain, $i$, is defined as the number of LAs in the same MSC that the MT has passed by. The state transition rate $s_{i, i+1}=\lambda$ represents the MT moving rate from state $i$ to state $(i+1)$ to the neighbouring LA in the same MSC. The transition rate that the MT moving (from state $i$ to state 0 ) to another LA out of the MSC, denoted by $t_{i, 0}(i \geq 0)$, is $\mu$.

We assume $p_{i}$ to be the equilibrium state probability of state $i$. The expression for $p_{i}(i \geq 0)$ in term of $p_{0}$ is

$$
p_{1}=[\lambda /(\lambda+\mu)]^{\prime} p_{0}=\left(1-p_{0}\right)^{\dagger} p_{0}
$$

Using the law of total probability, the equilibrium state probability of state 0 is obtained as

$$
p_{0}=\frac{\mu}{\lambda+\mu}=1-\frac{\lambda}{\lambda+\mu}=1-\rho=\frac{r_{L 4}}{r_{M \triangle C}} \text {, with } \rho=\frac{\lambda}{\lambda+\mu}
$$




\subsection{Cost Analysis}

The location tracking cost is divided into two components: (1) the update cost, $U_{x}$, which incurred in completing the location update procedure, (2) the searching cost, $S_{x}$ which incurred in completing the call delivery procedure ( $x \in\{H L D$-only, $S R, S R C\}$ ). We consider communication (routing, sending, receiving messages) and database processing as our basic measures of cost. The protocols involve the setup of dedicated channel and the call delivery procedure includes a paging procedure for all schemes, thus we ignore these costs for all calculations. Some costs and parameters used to analyse the analytic model are summarized in Table 1.

Table 1. List of cost parameters

\begin{tabular}{|l|l|c|}
\hline Parameters & \multicolumn{1}{|c|}{ Meaning } & Value \\
\hline$D_{h}$ & The cost for a query or an update of the HLD & $\alpha D_{c v}$ \\
\hline$D_{c v}$ & The cost for a query or an update of the CLD/VLD & $\gamma N_{l o}$ \\
\hline$N_{r e}$ & $\begin{array}{l}\text { The cost for a signalling message through the re- } \\
\text { mote link to another MSC }\end{array}$ & $\beta N_{l o}$ \\
\hline$N_{l o}$ & $\begin{array}{l}\text { The cost for a signalling message through the local } \\
\text { link within the MSC }\end{array}$ & 1 \\
\hline$C_{\text {intra }}$ & The cost of an intra-MSC location update procedure & $2 N_{l o}+D_{c v}$ \\
\hline$C_{\text {inter }}$ & The cost of an inter-MSC location update procedure & $N_{l o}+4 N_{r e}+D_{c v}+D_{h}$ \\
\hline
\end{tabular}

\subsubsection{Update Cost}

According to the location update protocol for the intra-MSC and interMSC cases in Figure 3, the expression of the average movement costs for SR and SRC schemes are given by

$$
U_{S R}=U_{S R C}=p_{o} C_{\text {inter }}+C_{\text {ine } r a} \sum_{k=1}^{\infty} k p_{k}=(1-\rho) C_{\text {inter }}+\frac{\rho}{1-\rho} C_{\text {in } r a}
$$

The update cost for the HLD-only scheme is simply received by

$$
U_{H L D-\text { only }}=\left(2 N_{r e}+D_{h}\right)\left[(1-\rho)+\frac{\rho}{1-\rho}\right]
$$

\subsubsection{Searching Cost}

The searching cost for HLD-VLD and VLD-CLD depends upon the probability of a calling and called parties are in the same MSC, $q$, the cachehit ratio, $\delta$, as well as the cost of querying the pointed VLD that store in the 
cache CLD. The cache-hit ratio is defined as the relative frequency with which the cached pointer correctly points to the user's location when it is consulted [6]. Table 2 summarises all possible events, their corresponding probabilities, and costs for the searching procedures of all the schemes.

Table 2. Searching activities, probabilities and costs

\begin{tabular}{|c|c|c|c|c|c|}
\hline Scheme & \multicolumn{2}{|c|}{ Description } & Probability & Symbol & Cost \\
\hline HLD-only & \multicolumn{2}{|c|}{ - } & 1 & $\widetilde{S_{H L D-o n l_{y}}}$ & $3 N_{r e}+D_{h}$ \\
\hline \multirow{2}{*}{$\begin{array}{c}\text { SR } \\
\text { (HLD-VLD) }\end{array}$} & \multicolumn{2}{|l|}{ local call } & $p_{S R, 1}=q$ & $S_{l, S R}$ & $2 N_{l o}+D_{c v}$ \\
\hline & \multicolumn{2}{|c|}{ remote call } & $p_{S R, 2}=(1-q)$ & $S_{2, S R}$ & $2 N_{l o}+3 N_{r e}+2 D_{c v}+D_{h}$ \\
\hline \multirow{3}{*}{$\begin{array}{c}\text { SRC } \\
\text { (VLD-CLD) }\end{array}$} & local call & & $p_{S R C, 1}=q$ & $S_{I, S R C}$ & $2 N_{l o}+D_{c v}$ \\
\hline & \multirow{2}{*}{$\begin{array}{l}\text { remote } \\
\text { call }\end{array}$} & hit & $p_{S R C, 2}=\delta(1-q)$ & $S_{2, S R C}$ & $2 N_{l o}+2 N_{r e}+2 D_{c v}$ \\
\hline & & miss & $p_{S R C, 3}=(1-\delta)(1-q)$ & $S_{3, S R C}$ & $3 N_{l o}+3 N_{r e}+3 D_{c v}+D_{h}$ \\
\hline
\end{tabular}

The average cost per unit time can be expressed as the product of the cost and the rate of their occurrence as

$$
S=\lambda_{c} \sum_{i} p_{i} S_{i}=\lambda_{c} S_{x}
$$

The total cost per unit time is the combined cost of location update and searching, denoted as $\mathrm{T}$, which is the sum of the two costs, given as

$$
T=U+S=\lambda_{m} U_{x}+\lambda_{c} S_{x}
$$

\subsection{Comparative Results}

In evaluating and comparing the cost of these strategies, we consider the call to mobility ratio $(C M R)$ as the ratio of the call arrival rate to the mobility rate and the cache-hit ratio as

$$
C M R=\frac{\lambda_{c}}{\lambda_{m}}=\frac{\delta}{1-\delta}
$$

In order to be able to estimate the total cost per unit time, the call arrival rate $\lambda_{c}$, and the rate at which the MT moves between LA, $\lambda_{m}$, are needed. We use the call to mobility ratio $(C M R)$ to study the performance of these schemes and compare the cost of the strategies by the ratio of the total costs $(T C R)$ described as follows

$$
T C R=\frac{T}{T_{\text {ref }}}=\frac{U_{x}+C M R^{*} S_{x}}{U_{\text {ref }}+C M R^{*} S_{\text {ref }}}
$$


For the analytical results given in this section, it is assumed that the cell is about $150-200 \mathrm{~m}$ of radius, whereby LA and MSC cover around 100 cells and 100 LAs, respectively. From equations (5) and (6), we get cost reduction of 10 times for the location update procedure referred to the HLD-only scheme. The methodology of evaluation used is to establish a common unit of measure for all cost terms, for example time delay. We use the sets of values of coefficient parameters $\alpha, \beta$, and $\gamma \in(1,2,3)$ as shown in Table 3 . The value of $N_{b o}$ is normalized to one since it can be seen as the lowest among the other costs. Parameter sets 6 and 8 capture the cases when it is significantly more expensive accessing the $D_{h}$ than $D_{c v}$. Figure 7 plots the variation of the normalized total cost with a wide range of $C M R(0.01$ to 100$)$. We used the value of $q=0.25$ so that CMR gets a threshold for caching of being beneficial [6]. It can be seen that (1) the cost improvement of the VLD-CLD/HLDVLD schemes have meaning only if the access cost to HLD is greater than that to VLD/CLD; (2) the VLD-CLD/HLD-VLD schemes always result in higher performance than the HLD-only strategy; (3) the VLD-CLD performs better than the HLD-VLD for a very wide range of call-to-mobility ratio, especially when CMR is greater than one.

Table 3. Coefficient parameters

\begin{tabular}{|c|c|c|c|c|c|c|c|c|c|}
\hline Class & $N_{l o}$ & $N_{r e}$ & $D_{c v}$ & $D_{h}$ & Class & $N_{l o}$ & $N_{r e}$ & $D_{c v}$ & $D_{h}$ \\
\hline 1 & 1 & 1 & 1 & 1 & 5 & 1 & 2 & 2 & 4 \\
\hline 2 & 1 & 2 & 1 & 1 & 6 & 1 & 2 & 2 & 6 \\
\hline 3 & 1 & 2 & 1 & 2 & 7 & 1 & 3 & 3 & 6 \\
\hline 4 & 1 & 2 & 2 & 2 & 8 & 1 & 3 & 3 & 9 \\
\hline
\end{tabular}

\section{CONCLUSION}

In this paper, the next generation mobile communication nctwork is proposed to integrate with a TINA-compliant architecture enabling to handle that kind of mobile-specific processes. This paper addresses design, modelling and comparison of a distributed location management. In the proposed network model, three mechanisms have been applied: distributed home location database, caching and replication strategy for handling the location management procedures. We use the local cache (CLD) and replication (VLD) location databases to reduce network signalling traffic and database updating as well as querying delay during location registration and call delivery procedure. The numerical results show that both strategies, VLD-CLD and HLD-VLD, can significantly reduce the total cost compared to the HLDonly scheme. Results also show that the replication with caching strategy performs better than the replication strategy. This paper also presents a simplc methodology for evaluating some basic location management algorithms 
for distributed location databases based on a TINA-compliant architecture for wireless communication networks.

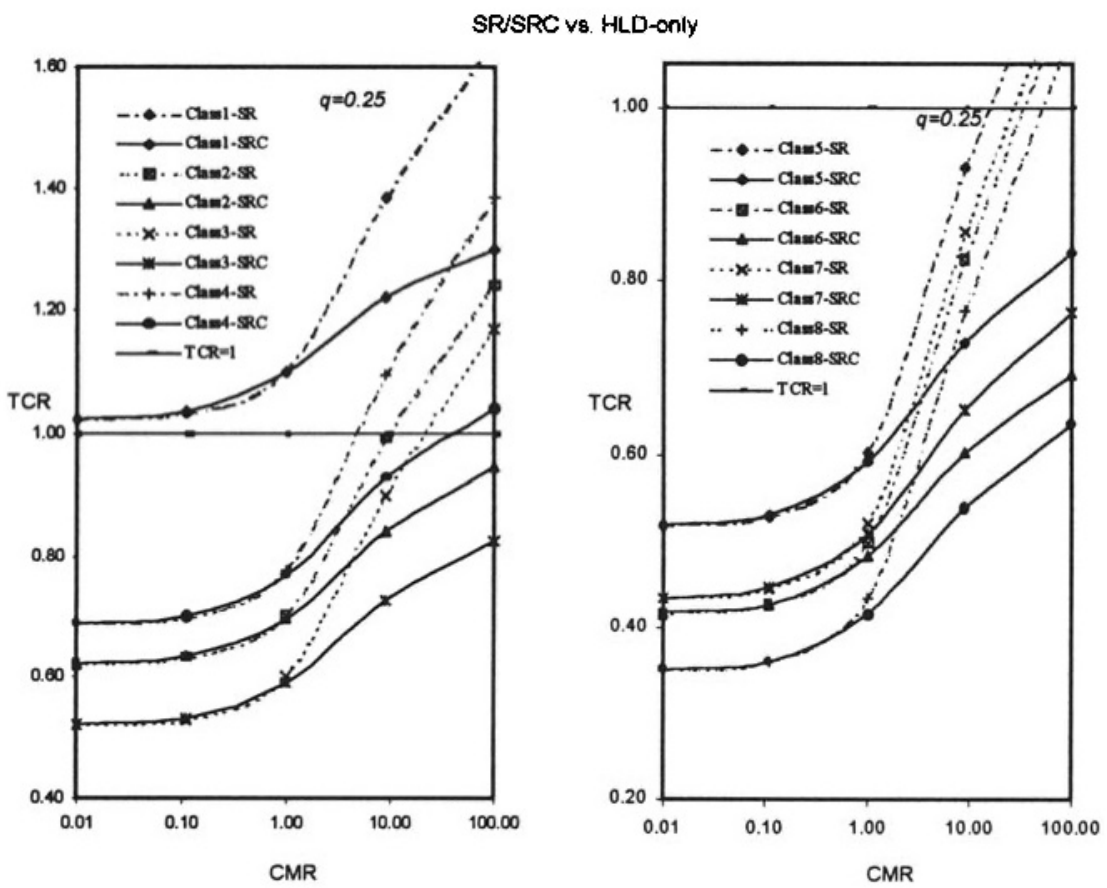

Figure 7. Total cost Ratio (TCR)

\section{REFERENCES}

[1] I.F. Akyildiz, J. McNair, J.S.M. Ho, H. Uzunalioglu, and W. Wang, "Mobility Management for Next Generation Wireless Systems", Proc. IEEE, Vol. 87, No. 8, Aug. 1999, pp. 1347-1384.

[2] C. Eynard, M. Lenti, A. Lombardo, O. Marengo, and S. Palazzo, " $A$ methodology for the performance evaluation of data query strategies in UMTS", IEEE JSAC, Vol. 13, No. 5, June 1995, pp. 893-907.

[3] B.C. Kim, J.S. Choi, C.K. Un, "A new distributed location management algorithm for broadband Personal Communication Networks", IEEE Trans. on Vehicular Technology, Vol. 44, No. 3, Aug. 1995, pp. 516-524.

[4] K. Wang, J. Huey, "A cost effective distributed location management strategy for wireless networks", Wireless Networks, Vol. 5, No. 4, Aug. 1999, pp. 287-297.

[5] N.M. Hoang, H. R. van As, "Connection and Location Management based on a TINA-compliant Architecture for UMTS", Smartnet'99, Thailand, Nov. 1999.

[6] R. Jain, Y.-B. Lin, C. Lo, S. Mohan, "A Caching strategy to reduce Network impact of PCS", EEE JSAC, Vol. 12, No. 8, Oct. 1994, pp. 1434-1444.

[7] J. Schuringa, "Performance Modeling of Location Management Strategies in Mo bile Networks", Master Thesis 1995, Department of CS, Univ. of Twente. 